\title{
Development of Microstrip Patch Antenna Design for GPS in Myanmar
}

\author{
Zin Mar Phyo, Tint May Nway, Khin Kyu Kyu Win, Hla Myo Tun \\ Department of Electronic Engineering, Yangon Technological University, Gyogone, Insein Yangon, Republic of the Union of Myanmar
}

\section{Email address:}

hlamyotun.ytu@gmail.com (H. M. Tun)

\section{To cite this article:}

Zin Mar Phyo, Tint May Nway, Khin Kyu Kyu Win, Hla Myo Tun. Development of Microstrip Patch Antenna Design for GPS in Myanmar. American Journal of Electromagnetics and Applications. Vol. 8, No. 1, 2020, pp. 1-11. doi: 10.11648/j.ajea.20200801.11

Received: January 14, 2020; Accepted: January 27, 2020; Published: February 10, 2020

\begin{abstract}
Polarization matching between the transmitter and receivers is an important thing due to the signal losses and multipath fading. In GPS application, L1 Band is commonly used to find the location all over the world. In this research, edgetruncated circularly polarized microstrip patch antenna and slot-loaded circularly polarized microstrip patch antenna are presented. The first design is diagonally fed with a single coaxial probe and the upper edges are truncated to reduce the patch size. This design provides the bandwidth of $79.5 \mathrm{MHz}$ with the axial ratio bandwidth of $26 \mathrm{M} \mathrm{Hz}$. In the second design, the slots are loaded to the patch in order to reduce patch size. The single coaxial diagonal feed is also applied to the patch. This design achieves the bandwidth of $56 \mathrm{MHz}$ and the axial ratio bandwidth is about $22 \mathrm{M} \mathrm{Hz}$. The second design can also provide the size reduction of $18.16 \%$. However, the gain of the second antenna decreases to $3 \mathrm{~dB}$ due to its reduced size. As these simulated results, these two designs can be used in L1 Band GPS application. And then, these antenna designs are fabricated and measured the results such as return loss and axial ratio. The characteristics of these fabricated antennas are also applicable for the targeted application.
\end{abstract}

Keywords: Microstrip Patch Antenna, GPS, Electromagnetic Field, Fabrication, Measurement

\section{Introduction}

In the design of global positioning system and modern wireless communication, miniaturization of the antenna is one of the most critical components because GPS receiver antennas are usually mounted on the roof of the car and handheld devices. Therefore, the first requirement for these antennas is to have a compact structure. Nowadays, there are many types of antennas to be used as GPS receiving antennas such as patch, helix, fractal element antenna, dipole, loop and Planar Inverted F antenna. Presently, helix and patch antennas are popular due to their design structures and specifications [1,2]. Moreover, there are many techniques to reduce the antenna size such as using high permittivity materials as the dielectric substrate, folded patch so called inverted-U shape, inverted-F shape, shorting pin between the patch and the ground plane, slot loaded on the ground plane and slot loaded on the patch [5-7].

The civilian signals transmitted from GNSS satellites are right-hand circularly polarized (RHCP). This circular polarization allows the arbitrary orientation of the receiving antenna. Moreover, it has the benefit of cross polarization rejection. Therefore, the second requirement for GPS application is to achieve the circular polarization. In mobile and portable communication where the devices frequently change their locations and orientations, it is impossible to match the orientation between the transmitter antenna and receiver antenna [6]. To achieve this polarization matching, the transmitter and receiver must have the same frequency range, axial ratio, spatial orientation and sense of polarization. Circularly polarized antennas are reconcilable with these requirements because they can reduce multipath effects and provide flexibility in the orientation angle between transmitting and receiving antennas, thus circularly polarized antennas are widely used in mobile and wireless communication such as global navigation system and satellite communication system [9]. Moreover, for GPS antenna, it is required to have wide beam widths at both the azimuth plane and the elevation plane since the antennas have to receive radiating signals from at least four satellites at any one time to predict exactly the locations and directions on the ground $[2,8]$. 
In this research, designing the circularly polarized microstrip antenna for GPS application is challenging in which it requires two steps. First step is to design the antenna which can operate in L1 band for GPS application. For second step, circular polarization is achieved by either introducing perturbation segments on the single radiating patch or feeding the antenna with external power divider which can produce equal magnitude and 90 degree out of phase. And then, antenna characteristics such as impedance bandwidth, $3 \mathrm{~dB}$ beam width, gain, axial ratio bandwidth, directivity are analyzed and carried out to develop the performance of the proposed antenna to meet the requirements for the target application area. Finally, the optimized antenna design is fabricated using Eleven Lab machine at YTU and measured the performance of this fabricated antenna with Vector Network Analyzer.

\section{Proposed Model}

Microstrip patch antenna in basic form consists of the main four components: metallic patch, dielectric substrate, ground plane and feeding structure. The metallic patch can be square, rectangular, strip, circular, triangular, elliptical, or any combination of these shapes. Mostly, rectangular and circular shapes are used because they are the easiest in analysis and fabrication [10-16].

And then, dielectric substrate layer is placed between the patch layer and the ground plane. There are a lots of substrate material in specifications to choose according to the applications. The higher the value of dielectric constant of substrate, the smaller the antenna's size. The substrates which are thin with high dielectric constant are preferable for applications in compact structure.

The ground plane is beneath the substrate layer. In some antenna's design, the perturbations can be placed on the ground to enhance the antenna performance, but the cross polarization to the backside can be produced.

The feeding techniques are also presented in chapter 3 . There are four popular types: microstrip feed, coaxial probe feed, aperture-coupled feed and proximity-coupled feed.

The input impedance of the antenna can be minimized by properly positioning the feed position. Without proper matching, there can be radiation loss between the transmission and receiver. The length of the patch (nonradiating edge) is typically $\lambda_{\mathrm{g}} / 2$ (where $\lambda_{\mathrm{g}}$ is the wavelength inside the dielectric substrate). This means that the higher the resonant frequency, the smaller the antenna's size in which the dielectric constant and thickness do not change [17-24].

Patches are usually manufactured by etching or metal deposition techniques on low dielectric substrate or ceramic substrate to enhance the performance of antenna. However, for GPS application, high dielectric material is used to load the patch and reduce the size. Ideally, the dimensions of the substrate and the ground plane should be several wavelengths long from the patch to achieve the better performance.

It is needed to achieve a circularly polarized microstrip antenna for all satellite communication, especially GPS application. The first step involves to operate the specified frequency bandwidth for GPS L1 band. In second step, circular polarization is achieved by either introducing the diagonal feed or a perturbation segment to a basic microstrip patch antenna.

\section{Design Consideration of Antenna I}

In this design, the rectangular patch shape is firstly chosen because of its easy fabrication and analysis. The copper is used as the conducting patch and the ground plane. And then, FR-4 substrate $\left(\varepsilon_{\mathrm{r}}=4.3\right)$ is placed between the patch and ground plane as dielectric substrate because it is low cost, available in market.

In this research work, CST studio suit simulation tool is used to design the antenna. This tool has been developed with Maxwell's equations easier to understand the performance of the antenna. However, in student version, the mesh count available for a solver is restricted, and then time domain and frequency domain solvers are included.

The first stage is to design a conventional rectangular microstrip patch antenna operating $1.575 \mathrm{GHz}$ (GPS L1 band) and coaxial probe feed is chosen as its feeding methodology because it is easy to impedance match. And then, circular polarization can take place when two modes are excited with equal magnitude and $90^{\circ}$ out of phase. To achieve this circular polarization, the patch is fed diagonally with proper position to be impedance matching.

Based on the transmission line model, the width and length of the patch can be approximately determined using Equation 1 to 5 . These equations can only evaluate the initial dimensions of the antenna. Therefore, the parametric study is done by adjusting the dimensions of antenna and feed point location to meet the characteristics of circularly polarized antenna for GPS application. In order to achieve circular polarization, diagonal feed technique should be used on the conventional microstrip patch antenna for the generation of the two degenerate modes.

Firstly, rectangular microstrip patch antenna is diagonally excited and then the length and width of the antenna is tuned to match with the impedance of the transmission line. After determining the dimensions, the feed point location is analyzed to get the axial ratio $<3 \mathrm{~dB}$. The location of the feed point dominates the performance of the circular polarization. The feed position (f) is done the parametric study to give the most acceptable axial ratio and return at the desired frequency.

$$
\varepsilon_{\mathrm{reff}}=\frac{\varepsilon_{\mathrm{r}}+1}{2}+\frac{\varepsilon_{\mathrm{r}}-1}{2}\left[1+12 \frac{\mathrm{h}}{\mathrm{W}}\right]^{-\frac{1}{2}}
$$

where, $\frac{\mathrm{W}}{\mathrm{h}}>1$

$\varepsilon_{\mathrm{r}}$ is the dielectric constant of the substrate

$\varepsilon_{\text {reff }}$ is the effective dielectric constant

$$
\Delta \mathrm{L}=0.412 \mathrm{~h} \frac{\left(\varepsilon_{\text {reff }}+0.3\right)\left(\frac{\mathrm{W}}{\mathrm{h}}+0.264\right)}{\left(\varepsilon_{\text {reff }}-0.258\right)\left(\frac{\mathrm{W}}{\mathrm{h}}+0.8\right)}
$$


where, $\Delta \mathrm{L}$ is the extension length on each side

$\mathrm{h}$ is height of the substrate

$\mathrm{W}$ is width of the patch

$$
\begin{gathered}
\mathrm{L}_{\text {eff }}=\mathrm{L}+2 \Delta \mathrm{L} \\
\mathrm{L}_{\text {eff }}=\frac{\mathrm{c}}{2 \mathrm{f}_{\mathrm{r}} \sqrt{\varepsilon_{\text {reff }}}} \\
\mathrm{W}=\frac{1}{2 \mathrm{f}_{\mathrm{r}} \sqrt{\mu_{0} \varepsilon_{0}}} \sqrt{\frac{2}{\varepsilon_{\mathrm{r}}+1}}=\frac{\mathrm{v}_{0}}{2 \mathrm{f}_{\mathrm{r}}} \sqrt{\frac{2}{\varepsilon_{\mathrm{r}}+1}}
\end{gathered}
$$

Figure 1 shows the edge-truncated rectangular patch antenna with diagonally feed to produce circular polarization. This causes splitting two orthogonal modes with the required $90^{\circ}$ phase shift. In this design, the dimensions of ground plane are $63 \mathrm{~mm}$ and $63 \mathrm{~mm}$ and the dimensions of the patch are $45.7 \mathrm{~mm}$ and $43 \mathrm{~mm}$, the thickness of the antenna with $1.575 \mathrm{~mm}$. The location of the feed is also determined at $\mathrm{f}=$ $11 \mathrm{~mm}$. The simulated results of edge-truncated rectangular patch antenna in CST simulation tool are also presented.

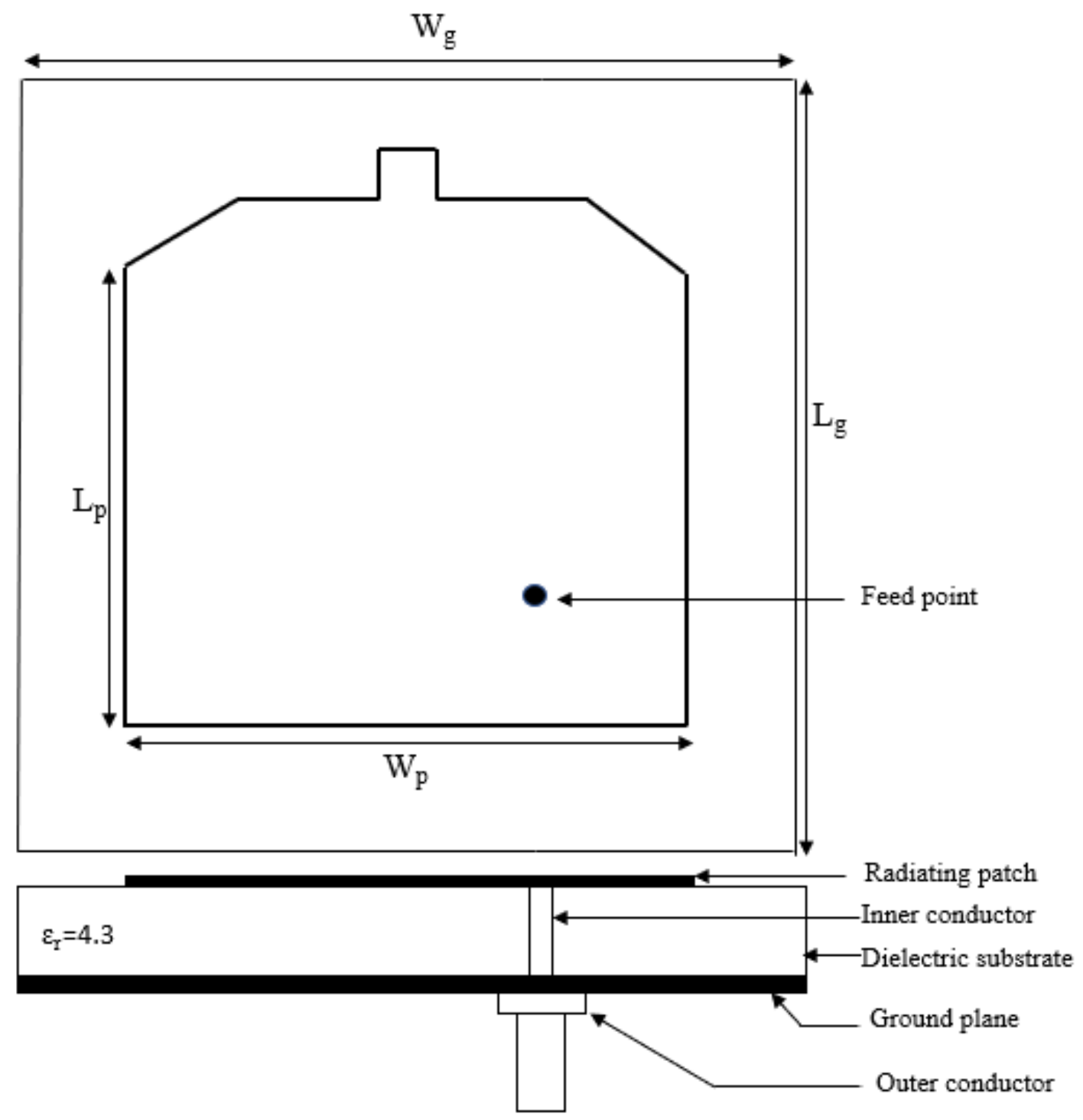

Figure 1. Geometry of Edge-truncated Rectangular Microstrip Patch Antenna.

The simulated return loss of the antenna is shown in Figure 2 and it is extended from 1.5405 to $1.62 \mathrm{GHz}$ (5.04\% with resonant frequency $1.5784 \mathrm{GHz}$ ). Return loss is the loss of power reflected back to the source in the transmission line. And then, it also presents to measure how well the antenna match with the transmission line. The high return loss indicates excellence in the matching condition between the microstrip antenna and the transmission line. In this case, the bandwidth is about $79.5 \mathrm{MHz}$ which is acceptable for GPS application. The smith chart plot is also shown in Figure 3 and shows the input impedance of the antenna at frequency $1.575 \mathrm{GHz}$. There is a small loop at the resonant frequency which indicates two resonance frequencies which are the characteristics of circular polarization. As shown in Figure 3, the impedance of the antenna achieves about $55 \mathrm{Ohms}$ at the desired frequency which is nearly match with the characteristic impedance. This impedance varies with the frequency. The overall impedance of the antenna provides about 51 Ohms. 


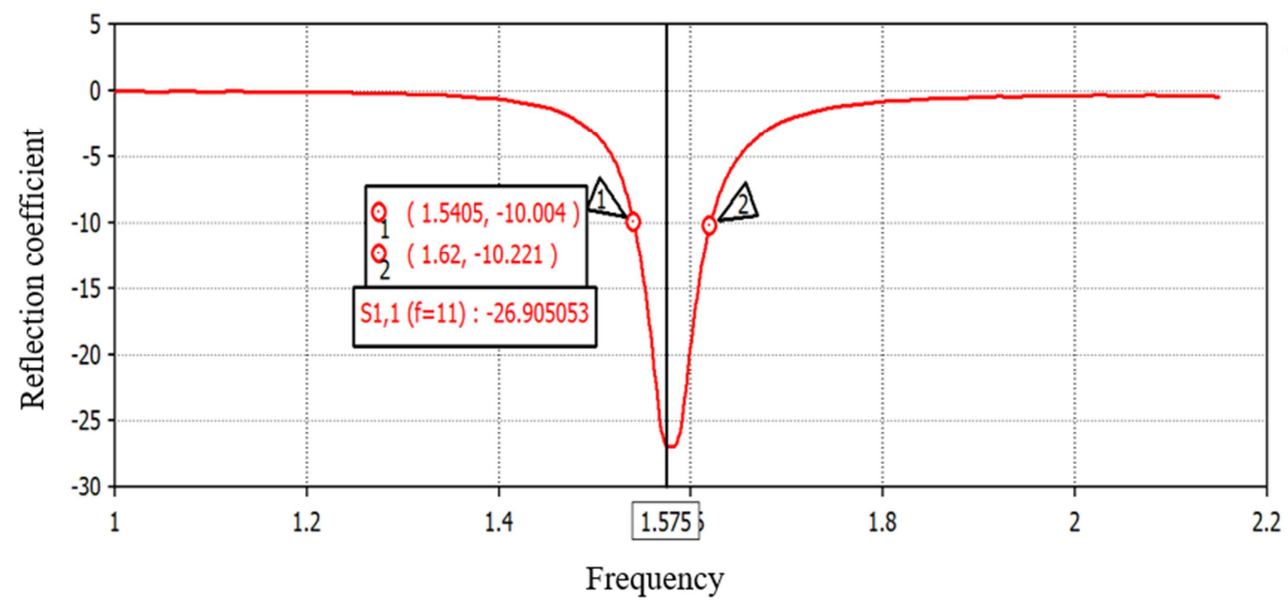

Figure 2. Graphical Result of Reflection Coefficient varying Overall Frequency.
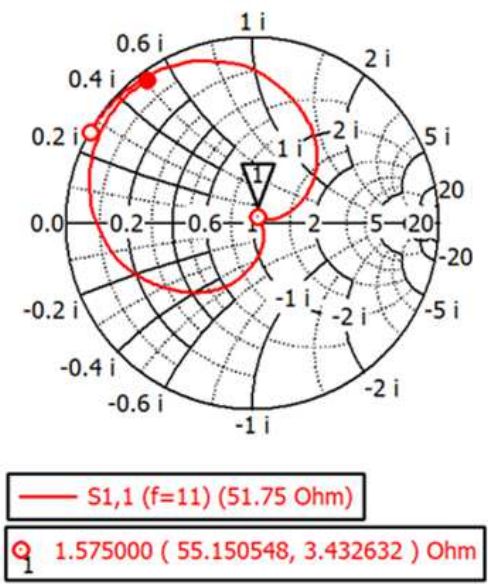

Figure 3. Smith Chart of Antenna showing Impedance at $1.575 \mathrm{GHz}$.

The value of axial ratio presents the characteristic of polarization in antenna. If the transmitter and receiver antennas are circularly polarized, they do not suffer the signal loss due to the polarization mismatch. The ideal value of axial ratio has $0 \mathrm{~dB}$ for circular polarization. The relation graph between the axial ratio, gain and frequency is shown in Figure 4.

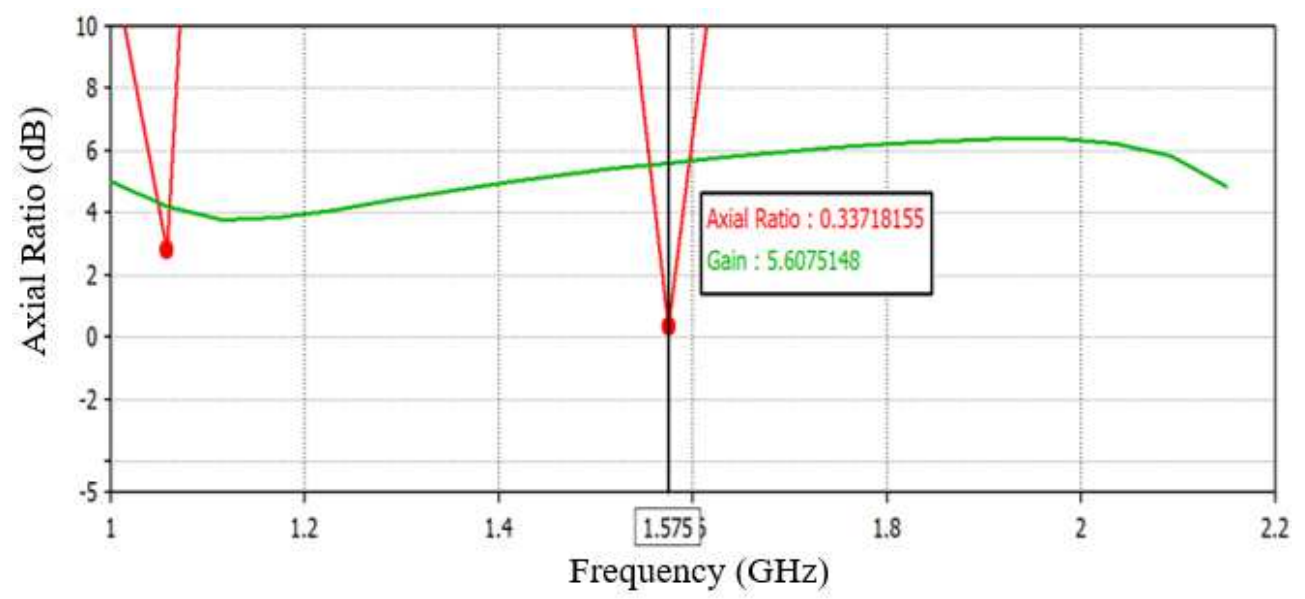

Figure 4. Graphical Results of Axial Ratio and Gain at Frequency $1.575 \mathrm{GHz}$.

The value of axial ratio must be less than $3 \mathrm{~dB}$ to be circularly polarized antenna. As shown in Figure 4, the axial ratio is lower than $3 \mathrm{~dB}$ in the frequency range $1.56 \mathrm{G} \mathrm{Hz}$ to
$1.586 \mathrm{G} \mathrm{Hz}$ which is a suitable range for GPS application. And then, in this figure, the directivity gain of the antenna is $5.6 \mathrm{~dB}$. However, the absolute gain is about $5.28 \mathrm{~dB}$ at 
$1.575 \mathrm{G} \mathrm{Hz}$. This result is slightly small, but the beam width of radiation pattern is large because it is inversely proportional to the antenna's gain. The circular beam width can be viewed in Figure 5. This value can be determined the width of the coverage area which has the circular polarization.

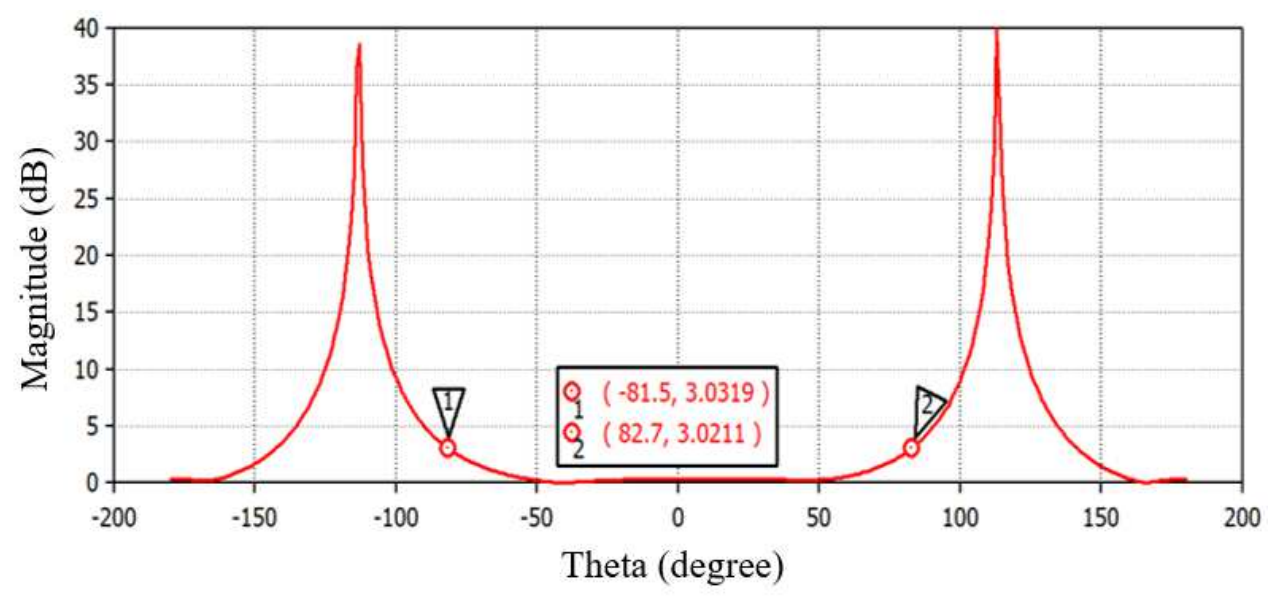

Figure 5. Axial Ratio Beam Width Result at $1.575 \mathrm{GHz}$ varying Theta $\left(\Phi=90^{\circ} \%\right.$.

According to this figure, the axial ratio bandwidth is about $164^{\circ}$ when the axial ratio is below $3 \mathrm{~dB}$. GPS receiver antennas are commonly used on the ground and they need to receive signal at least four satellites at once time. Therefore, its application requires broad beam width.

Moreover, to determine the type of circular polarization, the characteristic of surface current distribution has to be analyzed. It is shown in Figure 6 in which the surface current moves in counter clockwise direction at each interval $\pi / 2$. So, this antenna design processes Right Hand Circular Polarization (RHCP). If the feed position is changed, the type of polarization will be changed to Left hand Circular Polarization (LHCP).

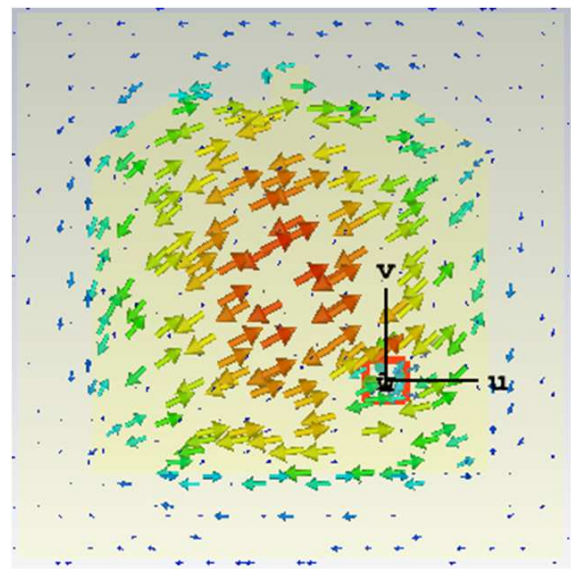

(a) Phase $0^{\circ}$

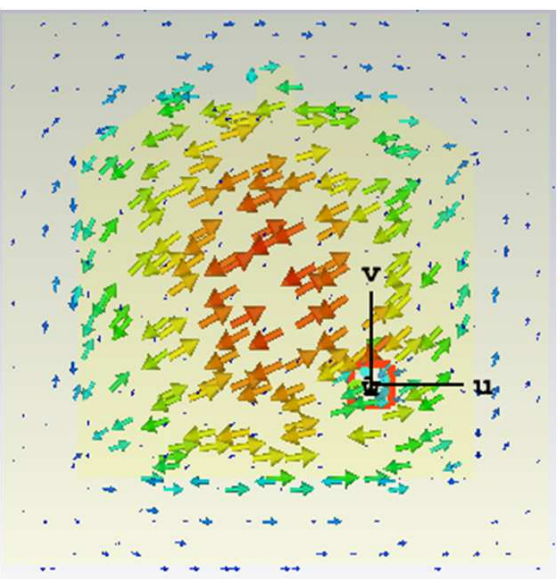

(c) Phase $180^{\circ}$

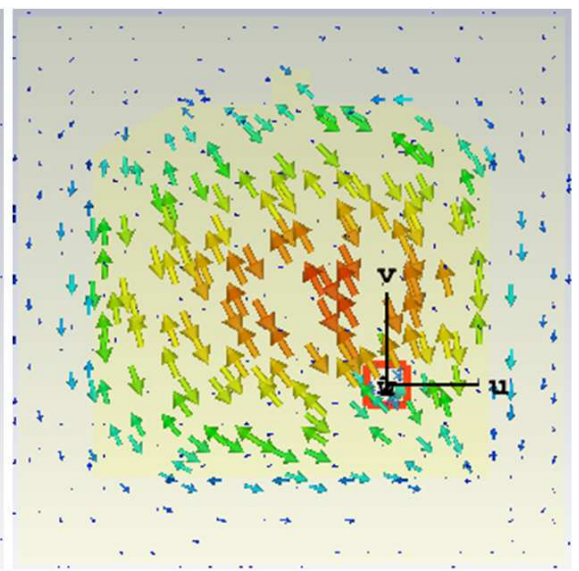

(b) Phase $90^{\circ}$

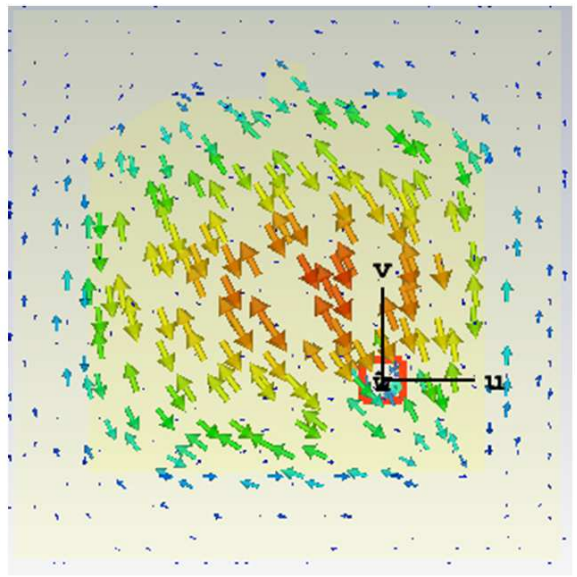

(d) Phase $270^{\circ}$

Figure 6. Surface Current Distribution of Antenna Design I. 
And then, the radiation pattern of the antenna should be analyzed. This figure is shown in Figure 7. From this figure (a), the maximum gain value of the antenna is found at 5.28 $\mathrm{dB}$ with $3 \mathrm{~dB}$ beam width of $96.8^{\circ}$ at $1.575 \mathrm{GHz}$ in the $\mathrm{XZ}$ plane. The maximum magnitude of the antenna can also be found $5.28 \mathrm{dBi}$ with $3 \mathrm{~dB}$ beam width of $101.1^{\circ}$ in the $\mathrm{YZ}$ plane.

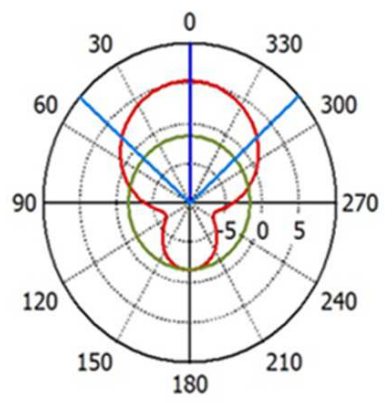

Theta / Degree vs dB

\begin{tabular}{|l} 
Frequency $=1.575 \mathrm{GHz}$ \\
Main lobe magnitude $=\quad 5.28 \mathrm{~dB}$ \\
Main lobe direction $=\quad 0.0 \mathrm{deg}$. \\
Angular width $(3 \mathrm{~dB})=96.8 \mathrm{deg}$. \\
Side lobe level $=-6.9 \mathrm{~dB}$
\end{tabular}

(a)

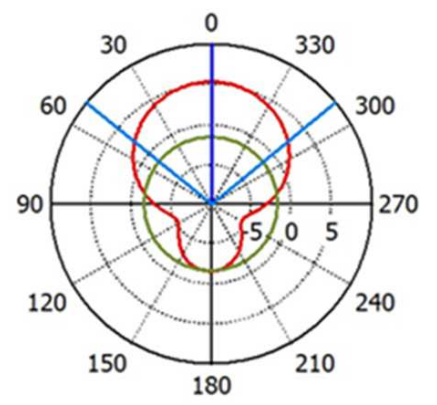

Theta / Degree vs. dB

Frequency = $1.575 \mathrm{GHz}$
Main lobe magnitude $=\quad 5.28 \mathrm{~dB}$
Main lobe direction $=\quad 0.0 \mathrm{deg}$.
Angular width $(3 \mathrm{~dB})=101.1 \mathrm{deg}$.
Side lobe level $=-6.9 \mathrm{~dB}$

(b)
Figure 7. Radiation Pattern for (a) $\phi=0^{\circ}, \theta$ varies $0^{\circ}$ to $180^{\circ}$ and (b) $\phi=$ $90^{\circ}, \theta$ varies $0^{\circ}$ to $180^{\circ}$.

The cross polarization of the antenna means the polarization which is orthogonal to the propagation direction. If the fields from the antenna are horizontally polarized, the cross polarization is vertically polarized. If the radiated fields from the antenna are RHCP, the cross polarization means LHCP. Therefore, the less the cross polarization, the better the radiation pattern of the antenna. There is no antenna which is $100 \%$ polarized in a single mode. The difference between co-polarization and cross-polarization should be 15 $\mathrm{dB}$ in some condition. The following Figure 8 shows the

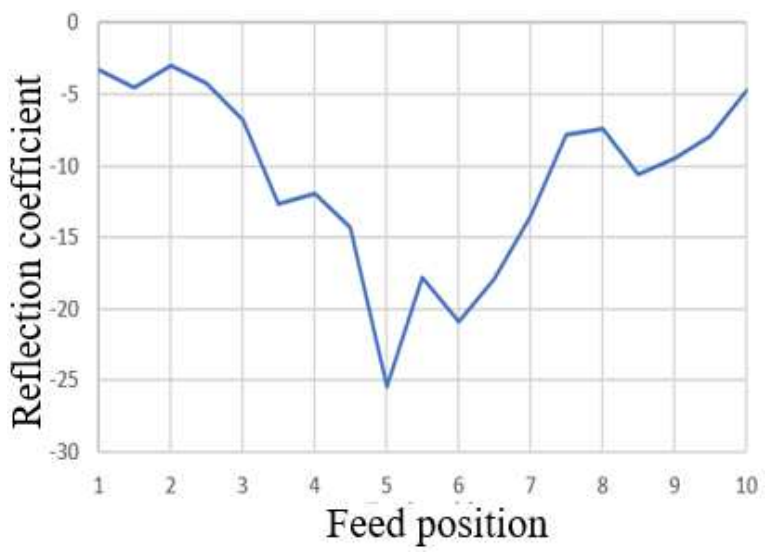

relation between co-polarization and cross-polarization of the antenna at $1.575 \mathrm{GHz}$ in both two planes.

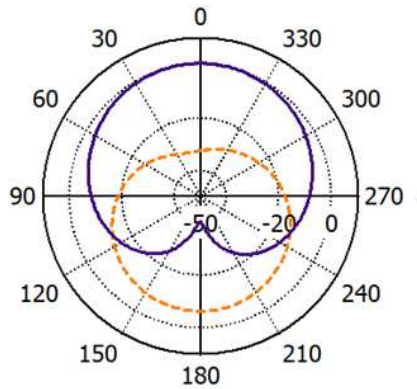

(a)

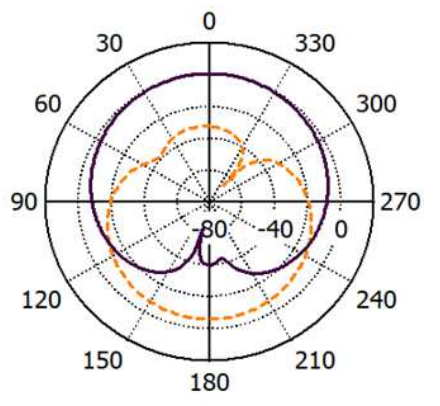

(b)

Figure 8. Relation Between Cross-polarization and Co-polarization (a) $\phi=$ $0^{\circ}, \theta$ varies $0^{\circ}$ to $180^{\circ}$ and (b) $\phi=90^{\circ}, \theta$ varies $0^{\circ}$ to $180^{\circ}$.

Consequently, the size of the antenna is reduced to be more flexible for GPS application. Among the methods of reducing the size of the antenna, the slots are placed to the radiating patch.

\section{Design Consideration of Antenna II}

In this design, the rectangular shaped antenna (ground's dimensions: $76 \times 67 \mathrm{~mm}$ ) is used as the initial design. In order to reduce the overall size of the antenna, the rectangular shaped slots are added at each four edges of the patch in which these four slots do not have equality in length. Moreover, the diagonal feeding is also excited as Antenna I in order to produce orthogonal electric fields which is the characteristic of the circular polarization. After perturbation step, the best feeding point is also analyzed by doing parametric study. When the feed location is changed (the values of dimensions are constant), the axial ratio and return loss extremely change. These relation graphs are illustrated in Figure 9.

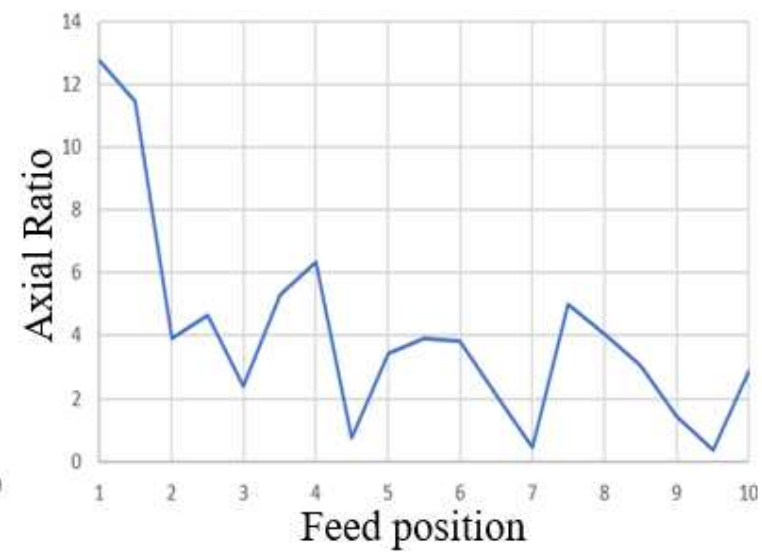

Figure 9. Parametric Analysis of Feed Location (f) at 1.575 GHz on Return Loss and Axial Ratio on Slot-loaded Rectangular Patch Antenna.

According to above figure, the location of the excitation relatively dominates on the performance of the antenna. The best reflection coefficient ( $\tau=-25.46)$ point can be found at the feed point ( $\mathrm{f}=5 \mathrm{~mm}$ ), but at that feed point, the value of 
axial ration is 3.422 which cannot match with circular polarization. And then, the best axial ratio $(A R=0.3562)$ point locates at the feed point $(f=9.5 \mathrm{~mm})$, however, the reflection coefficient is only $-7.9 \mathrm{~dB}$ at that feed point. So, this point cannot match with the transmission line with 50 Ohms.

Therefore, the most suitable feeding point is found out to be a compact circularly polarized antenna. For both impedance matching and circular polarization of the antenna, the feed location ( $\mathrm{f}=4.5 \mathrm{~mm}$ ) is the proper feeding point for L1 GPS application. The optimized antenna's design is illustrated in Figure 10. In this design, the current meanders through the patch due to the effect of loaded slots on the radiating patch.

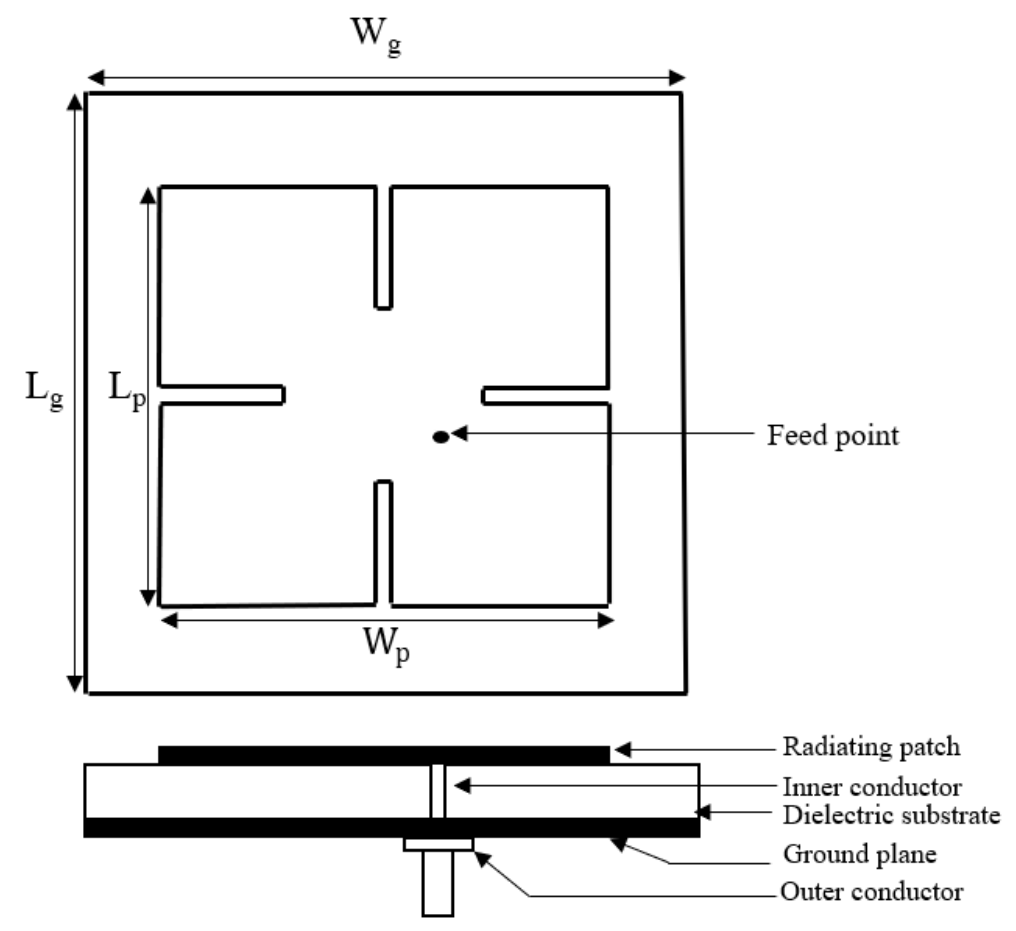

Figure 10. Geometry of Slot-Loaded Rectangular Microstrip Patch Antenna.

In this section, the simulated results of slot-loaded rectangular microstrip patch antenna are described and explained in details. Firstly, the relation between reflection coefficient and frequency is presented in Figure 11.

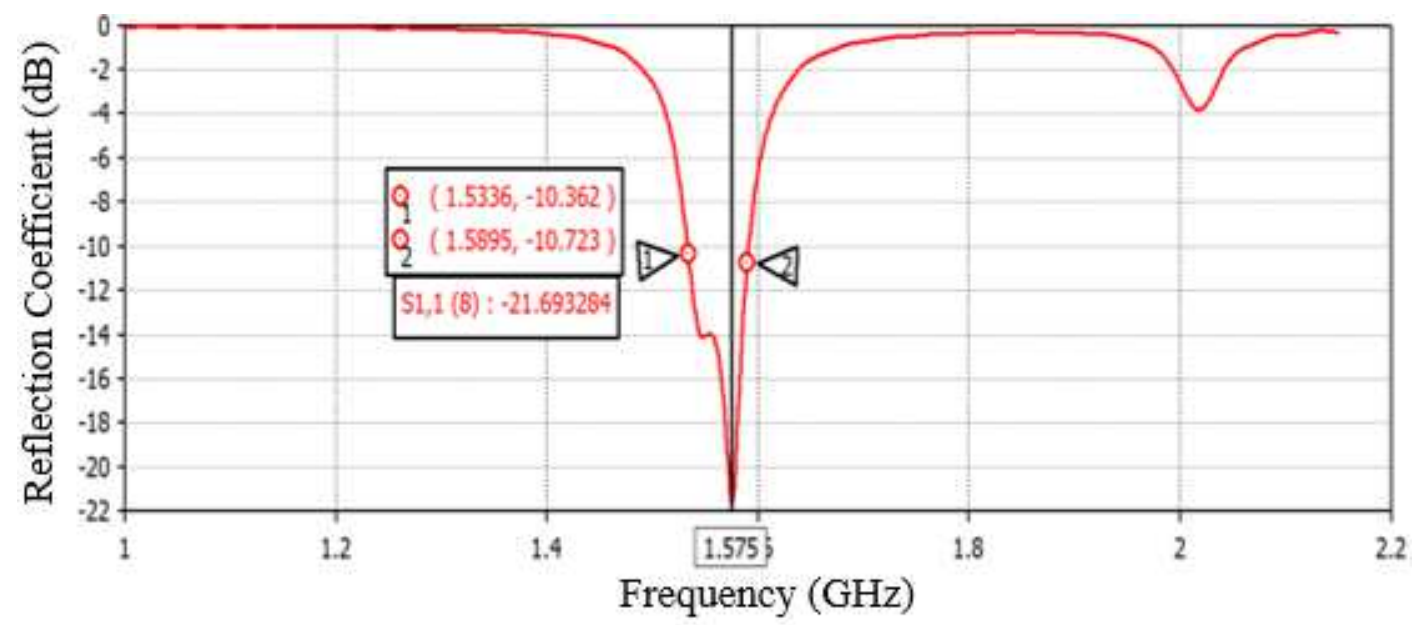

Figure 11. Graphical Result of Reflection Coe fficient varying Overall Frequency.

The return loss expresses the reflected power back to the transmission source. Therefore, the higher the magnitude of the return loss, the better the impedance matching of the antenna. Mostly, the amount of the reflection coefficient is defined below $-10 \mathrm{~dB}$ for matching condition. In this graph, the fractional bandwidth of the antenna is $3.55 \%$ in which the bandwidth extends from 1.5336 to $1.5896 \mathrm{GHz}(56 \mathrm{MHz})$. There is another parameter to check the impedance matching condition. This smith chart of the antenna can be found in Figure 12. In this smith chart, the impedance of the antenna is about $45 \mathrm{Ohms}$ at resonant frequency which is nearly same with the characteristic impedance of the transmission line. 
And then, there can be found small loop which indicates two resonant frequencies to generate two orthogonal electric fields. In order to produce circular polarization, there must have two electric fields which have equal magnitude and $90^{\circ}$ out of phase between them.

The magnitude of axial ratio responses the performance of circular polarization. In linear polarization, the magnitude of the axial ratio is infinite. However, the axial ratio of the circularly polarized antenna is generally defined below $3 \mathrm{~dB}$. For excellent circularly polarized antenna, the value of the axial ratio is less than $1 \mathrm{~dB}$. The axial ratio of the ideal $\mathrm{CP}$ antenna is $0 \mathrm{~dB}$. The GPS receiver antennas frequently change location on the ground. Therefore, they must be circularly polarized to immune the polarization mismatch and faraday's effect. The graph of axial ratio and directivity gain varying overall frequency is illustrated in Figure 13.

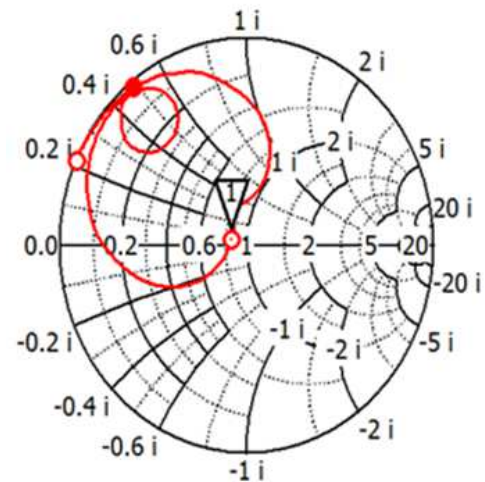

Q $1.575000(45.785061,2.325107)$ Ohm

Figure 12. Smith Chart of Antenna showing Impedance at $1.575 \mathrm{GHz}$

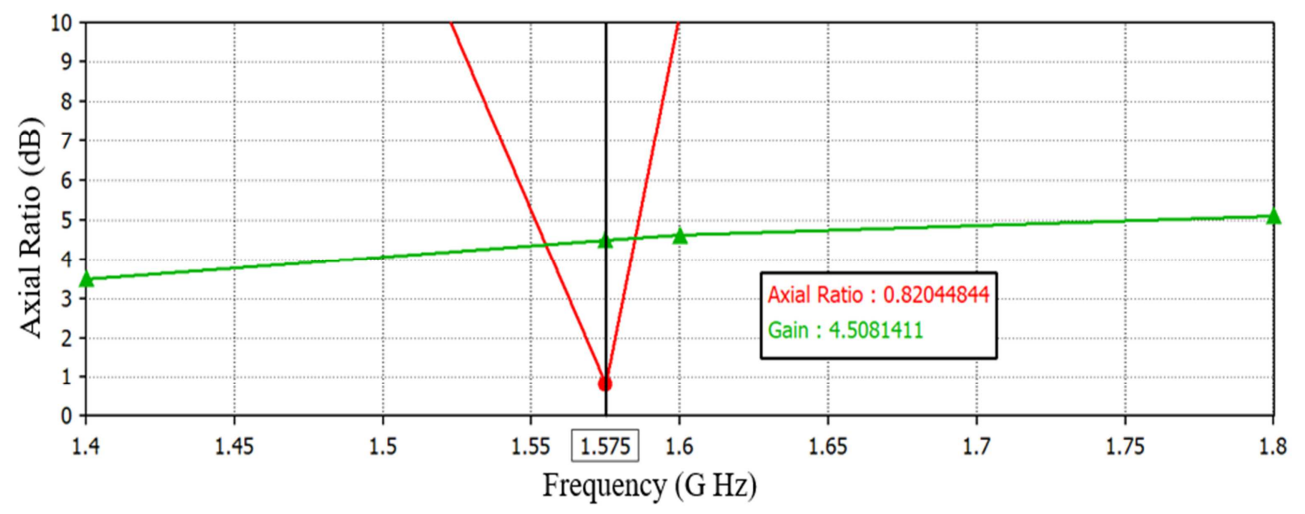

Figure 13. Graphical Results of Axial Ratio and Gain at Frequency $1.575 \mathrm{GHz}$.

As shown in Figure 13, the axial bandwidth of the antenna is in the frequency range of $1.56 \mathrm{GHz}$ to $1.582 \mathrm{GHz}$ where the value of axial ratio is lower than $3 \mathrm{~dB}$ that is the characteristic of circular polarization. The directivity gain of the antenna is about $4.51 \mathrm{~dB}$ in which it does not contain the matching loss, conductance loss and dielectric loss. And then, the absolute gain of this antenna is about $3.06 \mathrm{~dB}$ that is why the loss tangent of FR-4 substrate is relatively high. When the loss tangent of substrate is so high, the dielectric loss also increases. However, the beam width of radiation pattern is large because it is inversely proportional to the antenna's gain. The circular beam width can be viewed in Figure 14. This value can be determined the width of the coverage area which has the circular polarization.

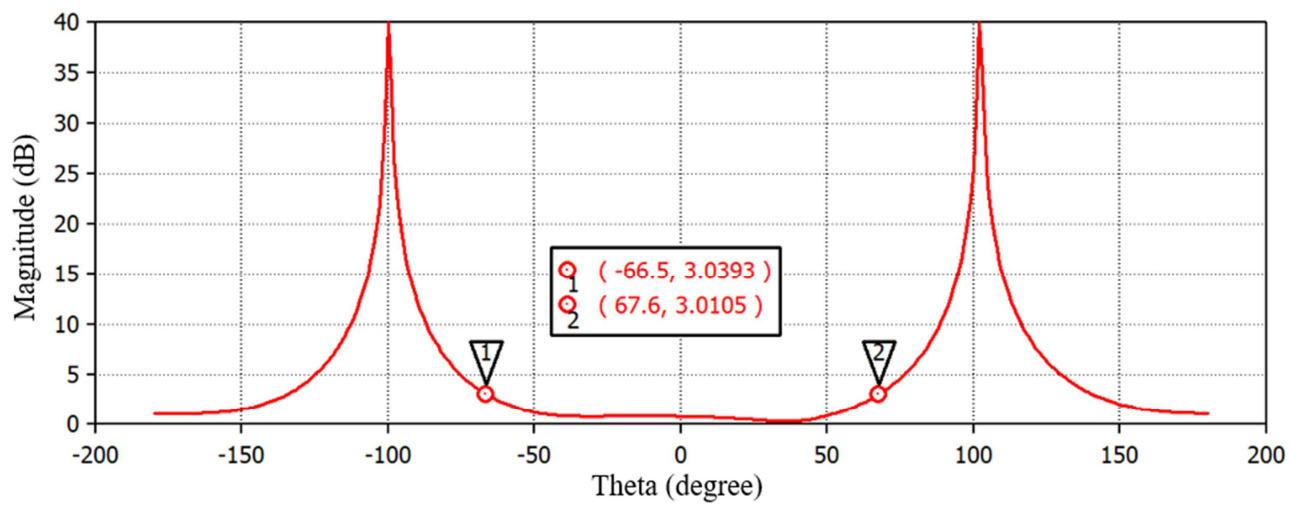

Figure 14. Axial Ratio Beam Width Result at $1.575 \mathrm{GHz}$ varying Theta (" $\Phi=90^{\circ}$ ”).

As shown in Figure 14, the axial ratio beam width is about $134^{\circ}$ which is axial ratio less than $3 \mathrm{~dB}$. The wider the beam width, the more signal can receive from the GPS satellite. Generally, at least four satellites are needed to receive the signal from the satellite at once time.

Moreover, GPS application commonly requires Right-hand Circularly Polarized microstrip patch antenna. Therefore, the direction of the surface current distribution is analyzed to 
distinguish the types of polarization such as LHCP and RHCP. The current distribution of the antenna which is separated the interval $\pi / 2$ is illustrated in Figure 15. In this figure, the magnitude of the current is relatively small because of its low gain. However, the surface current on the patch moves counter-clockwise direction. As this fact, this antenna can be said Right-hand circularly polarized antenna.

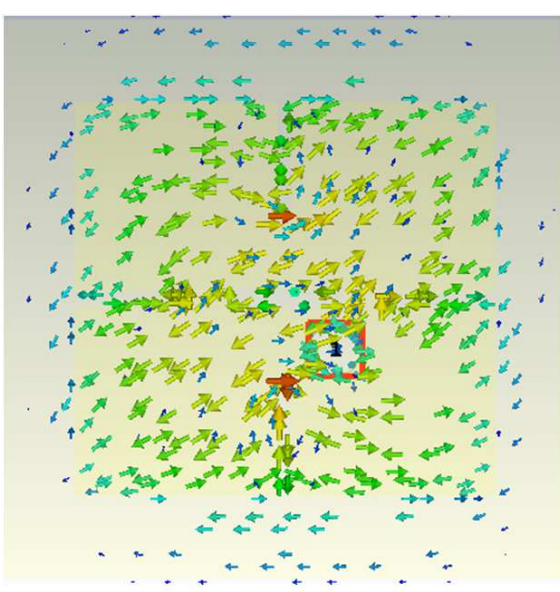

(a) Phase $0^{\circ}$

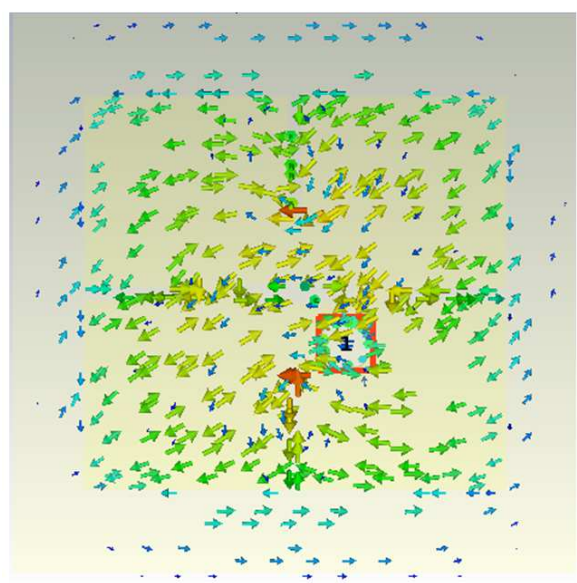

(c) Phase $180^{\circ}$

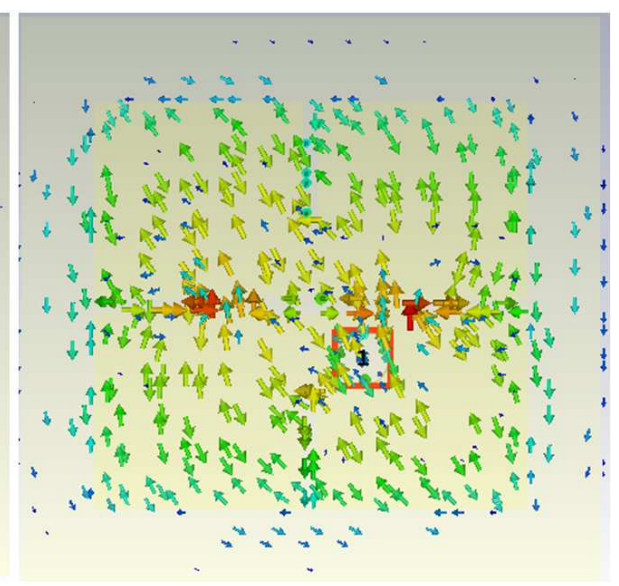

(b) Phase $90^{\circ}$

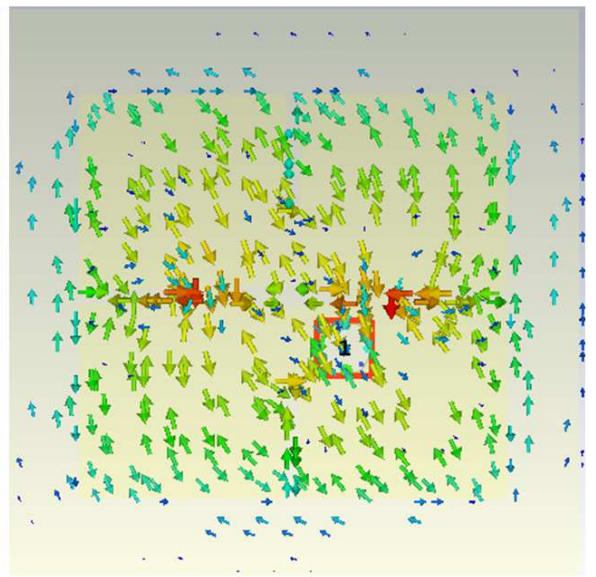

(d) Phase $270^{\circ}$

Figure 15. Surface Current Distribution of Antenna Design II.

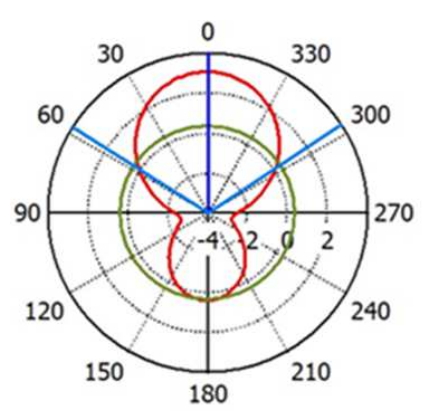

Theta / Degree vs. dB

\begin{tabular}{|l|} 
Frequency $=1.575 \mathrm{GHz}$ \\
Main lobe magnitude $=\quad 3.06 \mathrm{~dB}$ \\
Main lobe direction $=\quad 0.0 \mathrm{deg}$. \\
Angular width $(3 \mathrm{~dB})=113.8 \mathrm{deg}$. \\
Side lobe level $=\quad-2.7 \mathrm{~dB}$
\end{tabular}

(a)

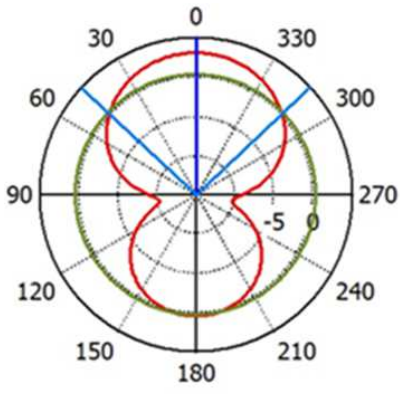

Theta / Degree vs. dB

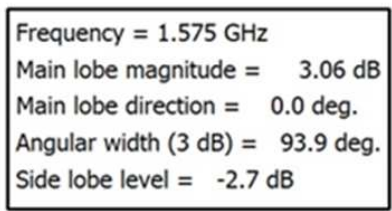

(b)

Figure 16. Radiation Pattern for (a) $\phi=0^{\circ}, \theta$ varies $0^{\circ}$ to $180^{\circ}$ and (b) $\phi=$ $90^{\circ}, \theta$ varies $0^{\circ}$ to $180^{\circ}$.

The radiation pattern is one of the most important parameters in the radiation part of the antenna. The types of radiation and how antenna receive or transmit can be distinguished by checking this radiation pattern. There are many types of radiation pattern such as omnidirectional, bi-directional and uni-directional. The radiation pattern with maximum magnitude of gain is presented in Figure 16. According to this figure, the maximum gain can be found $3.06 \mathrm{~dB}$ with beam width 113.8 degree in $\mathrm{XZ}$ plane and the maximum gain can also be found the same gain in $\mathrm{YZ}$ plane, however, the beam width is narrower than $\mathrm{XZ}$ plane.

Another parameter of the antenna is cross polarization which is unwanted signal. Generally, the magnitude of cross polarization should be less $15 \mathrm{~dB}$ than co-polarization. If so, the purity of the antenna's radiation can be received. The relation between the co-polarization and cross-polarization is illustrated in Figure 17 (a) XZ plane and (b) YZ plane. As shown in figure, this antenna design can relatively achieve low cross-polarization radiation. 


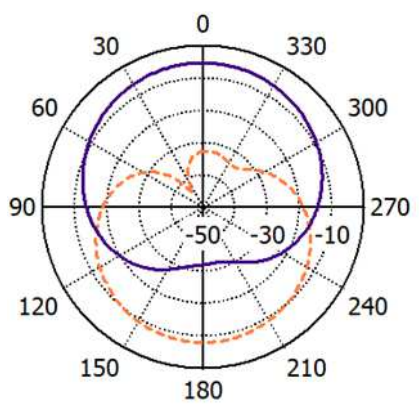

(a)

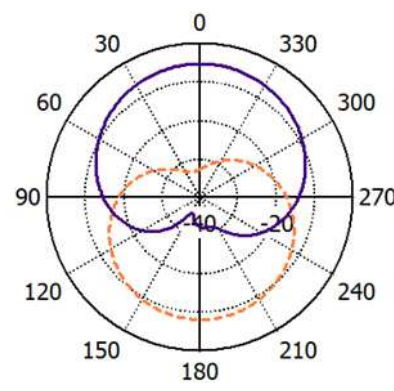

(b)
Figure 17. Relation Between Cross-polarization and Co-polarization (a) $\phi=$ $0^{\circ}, \theta$ varies $0^{\circ}$ to $180^{\circ}$ and (b) $\phi=90^{\circ}, \theta$ varies $0^{\circ}$ to $180^{\circ}$.

\section{Comparison Between Antenna Design I and Antenna Design II}

The implementation of design and simulated results in CST simulation tool are presented in above sections. There are good points and weak points of the input characteristic and radiation characteristic of design pattern in these two antenna designs. Table 1 describes the comparison between edge-truncated microstrip patch antenna and slot-loaded microstrip patch antenna.

Table 1. Comparison Between Edge-truncated Microstrip Patch Antenna and Slot-loaded Microstrip Patch Antenna.

\begin{tabular}{lll}
\hline Antenna Characteristics & $\begin{array}{l}\text { Edge-truncated } \\
\text { antenna }\end{array}$ & $\begin{array}{l}\text { Slot-loaded } \\
\text { antenna }\end{array}$ \\
\hline Reflection coefficient & $-26.9 \mathrm{~dB}$ & $-21.69 \mathrm{~dB}$ \\
Bandwidth & $79.5 \mathrm{MHz}$ & $56 \mathrm{MHz}$ \\
Axial Ratio & $0.337 \mathrm{~dB}$ & $0.82 \mathrm{~dB}$ \\
Axial Ratio Bandwidth & $26 \mathrm{MHz}$ & $22 \mathrm{MHz}$ \\
Directivity gain & $5.6 \mathrm{~dB}$ & $4.5 \mathrm{~dB}$ \\
Absolute Gain & $5.28 \mathrm{~dB}$ & $3.06 \mathrm{~dB}$ \\
Beam width & $96.8 \mathrm{degree}$ & $113.8 \mathrm{degree}$ \\
AR beam width & $101.1 \mathrm{degree}$ & $134 \mathrm{degree}$ \\
Patch dimensions & $45.7 \times 43 \mathrm{~mm}$ & $37.4 \times 35.1 \mathrm{~mm}$ \\
Ground dimensions & $63 \times 63 \mathrm{~mm}$ & $50 \times 50 \mathrm{~mm}$ \\
\hline
\end{tabular}

\section{Discussions}

In this research, two circularly polarized microstrip patch antennas are designed in CST simulation tool, fabricated using milling machine at YTU and finally measured the characteristics of these fabricated antennas using Vector Network Analyzer. These two antenna designs are analyzed on the characteristics of simulated and measured results. In Antenna design I (edge-truncated microstrip patch antenna), the dimensions of the ground plane are $63 \times 63 \mathrm{~mm}$ and that of the patch are $45.7 \times 43 \mathrm{~mm}$. The directivity gain of this design is about $5.6 \mathrm{~dB}$ and the absolute gain is $5.28 \mathrm{~dB}$. Therefore, the gain efficiency $\eta$ of $94.28 \%$ is resulted on this design. And then, the $3 \mathrm{~dB}$ beam width of 96.8 degree will also be found, thus this antenna design can receive signal from large coverage. The beam width with the axial ratio less than $3 \mathrm{~dB}$ have 101.1 degree. The impedance bandwidth and axial ratio bandwidth of this design also result $79.5 \mathrm{MHz}$ and
$26 \mathrm{MHz}$ respectively. These bandwidths are applicable for GPS L1 band application in which the specified bandwidth of the GPS application is at least $10 \mathrm{MHz}$. The magnitude of the return loss can be found about $-26.9 \mathrm{~dB}$ and the value of the axial ratio can also be found about $0.337 \mathrm{~dB}$ at resonant $1.575 \mathrm{GHz}$. In measured results, the magnitude of return loss is about $-24.9 \mathrm{~dB}$ at $1.575 \mathrm{GHz}$ and the frequency range extends from 1.54625 to $1.598 \mathrm{GHz}$ and from 1.667 to $1.7015 \mathrm{GHz}$. The dual resonant frequencies can be found in the fabricated antenna. The value of axial ratio frequently changes varying with overall frequency.

In Antenna design II (slot-loaded to the patch), the dimensions of ground plane are $50 \times 50 \mathrm{~mm}$ and that of the radiating patch are $37.4 \times 35.1 \mathrm{~mm}$. Therefore, the design II can be reduced in size $18.16 \%$ of the antenna design I adding slots to the patch. This result influences the gain of the antenna. By adding these slots, the size of antenna can be reduced, however, the gain of this design becomes low value. As this low gain, the radiation pattern becomes wider. The directivity gain and absolute gain can be achieved $4.5 \mathrm{~dB}$ and $3.06 \mathrm{~dB}$, thus the efficiency of this design has $68 \%$ because of spurious radiation of the patch. The $3 \mathrm{~dB}$ beam width is 113.8 degree and the axial ratio beam width is about 134 degree respectively. So, the beam width of design II processes the wider beam width. And then, the impedance bandwidth is about $56 \mathrm{MHz}$ and the axial ratio bandwidth can also be found about $22 \mathrm{MHz}$. Consequently, the magnitude of the return loss can be found $-21.69 \mathrm{~dB}$ and the value of axial ratio is $0.82 \mathrm{~dB}$ which meets the specification of circular polarization. In the measurement, the $-10 \mathrm{~dB}$ impedance bandwidth can be found from 1.59225 to $1.53475 \mathrm{GHz}$. And then, the value of axial ratio has less than $3 \mathrm{~dB}$ at resonant $1.575 \mathrm{GHz}$.

The main problem for this work is to find the suitable antenna design for GPS applications which would be affected to enhance the performance of the system. According to the analyses on antenna design I and II, the technical sound on antenna design II belongs to the high performance condition for GPS applications.

\section{Conclusion}

The GPS application requires the performance of circular polarization because this polarization can reduce the polarization mismatch and misalignment between the transmitting and receiving antennas. Consequently, GPS receiver antennas are impossible to constantly match with the polarization due to these antennas are mobile devices on the ground. This research work focused on designing circularly polarized microstrip patch antenna for GPS L1 band application. This paper presented two new designs based on the rectangular patch. The first antenna design is achieved by cutting the upper edges of the patch and adding the rectangular perturbation to reduce the overall size of the antenna. According the simulated results in CST simulation tool, the characteristics of this antenna design such as impedance bandwidth, the axial ratio bandwidth, gain and angular beam width meet the required specifications of GPS application. 
Therefore, this antenna design I can be used for GPS receiver antenna in the handheld devices in practice. In the measurement of fabricated antenna design I, the resonant frequency can be found at $1.575 \mathrm{GHz}$. Therefore, this fabricated antenna can also operate in GPS L1 Band application.

In second design, four slots are loaded to the edge of the patch and a central slot is added to reduce the patch size. The diagonal excitation technique dominates the performance of circular polarization. The characteristics of this simulated antenna design are suitable for the requirements of GPS application such as impedance bandwidth, the axial ratio bandwidth, gain and angular beam width. As these simulated results, this antenna design is applicable for GPS L1 band application. And then, the measured result of return loss also reconciles with GPS application by comparing with [5].

The antenna design II possesses better reducing size model than design I. However, the performance of design I is better than that of design II. The characteristics of antenna design II are also specified with the required facts of GPS receiver antenna. Therefore, the antenna design II is more suitable for handheld receiver device.

\section{Acknowledgements}

The author would like to thank many colleagues from the Antenna Engineering Research Group under the Department of Electronic Engineering of Yangon Technological University. The author acknowledges to Prof. Fujimoto of Nagasaki University for his kind guidance and supports for this research works.

\section{References}

[1] Levine, E. 2014. "A Review of GPS antennas." Consumer Electronic Times 3, no. 3 (July): 223-241.

[2] Anonymous. No Date. "GPS antennas." $2009 .<$ https://www.u-blox.com/ downloads/GPS antennas.pdf >

[3] Anonymous. No Date. "Global Positioning System." February 2019.

$<$ https://en.wikipedia.org/wiki/Global_Positioning_System >

[4] Anonymous. No Date. "GPS-Frequency-Bands." April 10, 2017. <http://www.everythingrf.com/community/gpsfrequency-bands $>$

[5] Devi, S. S., and Pradeepa, M. 2014. "Microstrip Inverted F antenna for GPS Application.” International Journal on Recent and Innovation Trends in Computing and Communication 2, no. 10 (October): 3143-3148.

[6] Kishore, M. R., Kumar, V. J., and Kumar, G. S. 2014. "Design \& Simulation of E-shaped Microstrip Patch Antenna for GPS Application.” International Journal of Engineering Research and Applications 4, no. 8 (August): 94-100.

[7] Holland, S. S. 2014. "Miniaturization of Microstrip Patch Antennas for GPS Applications". ME. Paper, University of Massachusetts Amherst.

[8] Gerald Moernaut, J. K., and Orban, D. No Date. "Basics of GPS antennas". The RF\& Microwave Solution Update
$<$ https://www.rfglobalnet.com/doc/basics-of-gps-antennas0001.pdf $>$

[9] Steven, S. G., Qi Luo, and Fuguo Zhu, 2014. Circularly Polarized Antennas. 3rd.ed. UK: John Wiley \& Sons, Ltd.

[10] Constantine, A. Balanis, 2005. Antenna Theory: Analysis and Design. 3rd.ed. Canada: John Wiley \& Sons, Ltd.

[11] Thomas, A. Milligan,. 2005. Modern Antenna Design. 2nd.ed. Canada: John Wiley \& Sons, Ltd.

[12] Hlassan, A., Elhefnawi, F., Sherbeni, Z.E., Moataza, H., and Elramly, S. 2010. "Compact Circularly Polarized Microstrip Array Antenna." Microwave and Optical Technology Letters 53, no. 3 (March): 604-609.

[13] Albon, E. and Cerretelli, M. 2005. "Microstrip Patch Antenna for GPS Application." Microwave and Optical Technology Letters 50, no. 3 (August): 1-4.

[14] Fujimoto, T., Yoshitake, Y., and Yagyu, D. 2015. "Design and Simulation Based Studies of a Dual-Band Circularly-Polarized Square Microstrip Antenna." Progress in Electromagnetics Research Letters 52, (March): 129- 134.

[15] Saravanan, M., and Rangachar, M. J. S 2018. "Design of Wide Beam Hexagonal Shaped Circularly Polarized Patch Antenna for WLAN Application." Proceedings of the 8th International Conference Soft Computing and Pattern Recognition (January): 1-9.

[16] Abdelaziz, A. Abdelaziz, and Dalia, M. Nashaat, 2007. "Compact GPS Microstrip Patch Antenna." IEEE 1, (July): 14.

[17] Patel, B. D., Narang, T., and Shubhangi Jain, 2013. "Microstrip Patch Antenna-A Historical Perspective of the Development." Conference on Advances in Communication and Control Systems (Novmember): 445-449.

[18] Orban, D. and Moernaut, G. J. K. 2009. "The Basics of Patch Antennas, Updated." September, 2009

$<$ https://www.orbanmicrowave.com/the-basic- of-patchantennas.pdf $>$

[19] Ramesh Garg, Prakash Bhartia, Inder Bahl, and Apisak Ittipiboon. 2001. Microstrip Antenna Design Handbook. 1st.ed. USA: Artech House antennas and propagation library.

[20] Abubakar Siddik, Mahabub Hossain, Dulal Haque, and Omar Faruque, 2019. "Design and Radiation Characterization of Rectangular Microstrip Patch Antenna for Millimeter-wave Communication," American Journal of Engineering Research 8, no. 1 (January): 318-324.

[21] Erik, O. Hammerstad. 1975. "Equations for Microstrip Circuit Design.” IEEE. (March): 268-272.

[22] Sunandan Bhunia, 2013. “Microstrip Patch Antenna's Limitation and Some Remedies." International Journal of Electronics \& Communication Technology 4, no. 1 (March): 38-39.

[23] Sourabh Bisht, Shweta Saini, Ved Prakash, and Bhaskar Nautiyal. 2014. "Study The Various Feeding Techniques of Microstrip Antenna Using Design and Simulation Using CST Microwave Studio." International Journal of Emerging Technology and Advanced Engineering 4, no. 9 (September): 318-324.

[24] Sunandan Bhunia, 2014. Microstrip Patch Antenna Design. 1st Edition. Germany: LAP LAMBERT Academic Publishing. 